\title{
S01-04
}

\section{ADULT ADHD: AN UNDER-DIAGNOSED CONDITION}

\section{C.N. Filipe}

CADIn - Centro de Apoio ao Desenvolvimento Infantil, Physiology Faculty of Medical Sciences U.N.L., Lisbon, Portugal

ADHD is a neurodevelopmental disorder that impairs executive functions, leads to maladaptive behavior and, in about $50 \%$, has a livelong persistence. Nevertheless, adult ADHD is a very much under-diagnosed condition.

We conducted a study aimed to identify ADHD in a special population of adults. Questionnaires were applied to the parents of children attending CADIn, a Clinical Centre for Developmental Disorders for evaluation or intervention (48\% of their children were diagnosed as having ADHD and $22 \%$ were diagnosed as having Pervasive Developmental Disorder). We expected a higher incidence of ADHD in this population as well as a better awareness for the condition.

We used the Adult ADHD Self-report Scale (ASRS-v1.1) and the questionnaires contained questions concerning degree of impairment, persistence of symptoms and comorbid psychiatric conditions. 114 parents were inquired, $29 \%$ fathers, $68 \%$ mothers $3 \%$ missed gender. $17 \%$ of the parents quoted above the cut-off-point for the ASRS-v1.1 screener and amongst those, $45 \%$ referred the ADHD symptoms as causing them severe impairment. Only 3 subjects had been previously diagnosed as ADHD. The prevalence of ADHD symptoms was similar for males and females. $56 \%$ of the ADHD population was diagnosed formerly as suffering from psychiatric disorder, compared to $35 \%$ of the total population. The more common diagnoses were depression (45\%) and anxiety $(30 \%)$.

We conclude that ADHD is much under-diagnosed in the adult population. Considering its high heritability, a closer evaluation of the parents of children diagnosed with ADHD is suggested. 